\title{
Injusticia de la desigualdad: factores determinantes en el Brasil, 1995 y 2009
}

\author{
Ana Claudia Annegues, Erik Alencar de Figueiredo \\ y Wallace Patrick Santos de Farias Souza
}

RESUMEN

Se analiza la evolución de la desigualdad injusta en el Brasil (1995-2009) mediante un enfoque no paramétrico de la estimación de la función de ingresos. Se utilizaron las medidas de entropía de Li, Maasoumi y Racine (2009) a fin de cuantificar separadamente las diferencias de ingresos para cada variable de esfuerzo. Se calculó un coeficiente de Gini de desigualdad injusta basado en los valores ajustados de la estimación no paramétrica, analizándose la robustez para las estimaciones, incluyendo variables de circunstancias. La evolución de las entropías mostró reducción de la diferencia de ingresos atribuida a la educación como factor determinante. Las variables de horas trabajadas y situación en el mercado laboral explican significativamente las diferencias de salarios imputadas al esfuerzo individual, pero la variable migratoria reveló escaso poder explicativo. Finalmente, el análisis de robustez demostró la plausibilidad de los resultados de cada etapa de la ejecución empírica.

PALABRAS CLAVE

CLASIFICACIÓN JEL
Condiciones económicas, distribución del ingreso, ingresos, educación, empleo, datos estadísticos, metodología estadística, Brasil

C14, D63

Ana Claudia Annegues es estudiante de doctorado del Programa de Postgrado en Economía de la Universidad Federal de Rio Grande do Sul, Brasil. annegues.ana@gmail.com

Erik Alencar de Figueiredo es profesor del Departamento de Economía del Programa de Postgrado en Economía de la Universidad Federal de Paraiba, Brasil. eafigueiredo@gmail.com

Wallace Patrick Santos de Farias Souza es estudiante de doctorado del Programa de Postgrado en Economía de la Universidad Federal de Rio Grande do Sul, Brasil. wpsfarias@gmail.com 


\section{I}

\section{Introducción}

La economía brasileña, marcada por un largo período de creciente desigualdad desde los años sesenta, registró una mejora de los indicadores sociales a partir de mediados de la década de 1990 (Azevedo, 2007), como resultado de un ambiente macroeconómico propicio para las transformaciones que se produjeron al estabilizarse la inflación e integrarse el Brasil en el mercado internacional. También es notorio que en ese período se incrementaron sustancialmente los programas de transferencia de renta gubernamental, lo que se tradujo en un aumento del nivel de ingresos y el consiguiente mejoramiento de los niveles de pobreza (Figueiredo y Netto Júnior, 2014).

No obstante, aunque esos indicadores podrían hacer creer que el Brasil se convirtió en un país más justo, en la bibliografía se ha cuestionado el tratamiento otorgado habitualmente a la desigualdad de ingresos, según el cual se considera a la igualdad perfecta como el ideal de justicia social. En ese sentido, es posible citar a autores muy diversos, desde los seguidores de la tradición rawlsiana, como Dworkin (1981) y Arneson (1989), que defienden las diferencias de ingresos vinculadas a preferencias individuales, hasta autores como Roemer (1998), que plantean el concepto de desigualdad de oportunidades.

Siguiendo este último enfoque, los resultados económicos individuales dependen de variables de responsabilidad (definidas como de esfuerzo) y de variables ajenas a la responsabilidad (definidas como de circunstancias). En esa línea de razonamiento, varios investigadores argumentan que solo la desigualdad debida a variables ajenas a la responsabilidad (también denominada desigualdad injusta) es socialmente indeseable.

Así, en algunos estudios se ha intentado medir la desigualdad de oportunidades y su contribución a la desigualdad total. Para ello, se han utilizado técnicas paramétricas directas e indirectas basadas en una forma funcional definida — como en Bourguignon, Ferreira y Menéndez (2007) — donde se abordan los determinantes de la distribución de ingresos contrafactual suponiendo

$\square$ Los autores agradecen el apoyo financiero del Consejo Nacional de Desarrollo Científico y Tecnológico (CNPq) (proyecto 470202/2012-2). que todos los individuos se encuentran en las mismas circunstancias, y en Salvi (2007), donde se exploran los datos en panel para distinguir las circunstancias y el esfuerzo en variables variantes e invariantes en el tiempo.

Aplicando el concepto de sensibilidad a la responsabilidad, Devooght (2008) mide la desigualdad de ingresos y un criterio de justicia calculado a partir de una función de ingresos $g$, con una aproximación mediante una regresión log-lineal. El autor solo utiliza variables de responsabilidad, ya que no siempre es posible obtener un conjunto bien definido de variables, en especial con respecto a los antecedentes familiares (nivel educativo y profesión de los padres), de modo que la parte explicada por las variables ajenas a la responsabilidad queda incluida en el término estocástico.

Sin embargo, esa metodología acarrea algunos problemas, en particular la endogeneidad generada por el grado de relación entre variables de esfuerzo y de circunstancias. Además, en muchos estudios, al suponerse una forma funcional para los ingresos individuales, puede incurrirse en un sesgo de especificación, dado el carácter aleatorio de las relaciones entre las variables.

En consecuencia, en este artículo se intenta cubrir esa laguna adoptando un enfoque no paramétrico ( $\sin$ forma funcional definida para $g$ ) con el objeto de responder a las siguientes preguntas, partiendo de la hipótesis de que los ingresos obedecen principalmente a las variables de responsabilidad: ¿cuál de ellas posee un mayor poder explicativo? ¿Han ido perdiendo influencia las variables de esfuerzo en la determinación de las diferencias de ingresos a lo largo del período analizado? Al suprimirse las variables ajenas a la responsabilidad, ¿no estarán expuestos los resultados a algún grado de sesgo?

Para ello se utiliza la siguiente estrategia empírica, compuesta de cuatro procedimientos. En primer lugar, se realizan análisis de especificación para justificar la utilización del método no paramétrico en la estimación de la ecuación de los ingresos. El segundo paso consiste en dividir la muestra en dos grupos de individuos, clasificados ad hoc como de "alto esfuerzo" y de "bajo esfuerzo", según cada variable de responsabilidad, y estimar los ingresos en relación con cada uno de los determinantes de esfuerzo (a la vez que los demás se mantienen constantes), observando su capacidad 
explicativa con respecto a la desigualdad de ingresos a lo largo del período analizado. La comparación entre las curvas se realiza utilizando como base las medidas de entropía tratadas en Racine (2006). En el tercer paso, se elaboran coeficientes de Gini de desigualdad injusta, a fin de medir la repercusión de las inferencias no paramétricas en el cálculo de los índices de justicia, y así verificar si el enfoque paramétrico tradicional tiende a sobrestimar o no esas medidas. Para realizar inferencias sobre los resultados obtenidos, en el cuarto y último procedimiento se efectúa un análisis de robustez empleando los datos de la Encuesta nacional de hogares (PNAD) de 1996. Se escogió ese año debido al suplemento de movilidad social asociado, en que se resumen los datos relativos a educación, escolaridad y ocupación de los padres de los individuos seleccionados (antecedentes familiares).

Se analiza el período comprendido entre 1995 y 2009, cuyos datos figuran en las PNAD anuales. Las variables de esfuerzo utilizadas son el nivel educativo, la decisión de emigrar, las horas trabajadas y la situación en el mercado laboral ${ }^{1}$. Como variable dependiente se emplea el logaritmo de los salarios nominales (variable sustitutiva del ingreso individual).

Además de esta Introducción, el resto del trabajo se estructura de la siguiente manera. En la sección II, que incluye los procedimientos metodológicos, se presentará la estrategia empírica que se divide en el análisis de la relación entre la desigualdad y el esfuerzo, la estimación de la variable sin forma funcional definida, y el modelo no paramétrico. La tercera sección está dedicada a la presentación de resultados y discusión, y la comparación de las distribuciones para cada variable de responsabilidad empleada y para la determinación de la medida de entropía. En la cuarta sección se presentan los debates sobre el tema y la quinta sección se reserva para las consideraciones finales.

\footnotetext{
${ }^{1}$ Esas variables se presentan de forma más detallada en la subsección 1.c de la sección II, dedicada al banco de datos.
}

\section{II}

\section{Procedimientos metodológicos}

El objetivo de esta sección es presentar los principales procedimientos metodológicos del estudio. En primer lugar, se destacan los conceptos teóricos vinculados a la bibliografía de la desigualdad de oportunidades o desigualdad injusta. A continuación, se enumeran los pasos de la ejecución empírica con el fin de aclarar los procedimientos de análisis del estudio (véase la subsección 1.a de la presente sección). Los métodos vinculados a cada paso se abordan en la subsección 1.b de esta misma sección. Finalmente, se presenta el banco de datos.

\section{Desigualdad de oportunidades: procedimientos teóricos y técnicos}

En el enfoque tradicional de la desigualdad de oportunidades se considera que el resultado económico de un individuo es fruto de factores circunstanciales, como los antecedentes familiares, la raza o el género, entre otros, así como del esfuerzo, vinculado a variables que pueden ser controladas por el interesado, entre las que se encuentran los años de estudio o las horas trabajadas, por ejemplo (Roemer, 1998) ${ }^{2}$. En resumen, a diferencia de la visión igualitaria moderna ${ }^{3}$, se considera justa una situación en que una sociedad garantiza a todos sus miembros, independientemente de sus circunstancias, el mismo acceso a las prestaciones sociales, de manera que el esfuerzo sea el responsable de que el acceso a esas prestaciones se transforme en ganancias reales para los individuos. Dicho de otra forma, solo la desigualdad resultante de variables circunstanciales es socialmente indeseable. Por lo tanto, igualar las oportunidades significa corregir las circunstancias desiguales y mantener inalteradas las diferencias de esfuerzo ${ }^{4}$.

\footnotetext{
2 Alternativamente, la desigualdad de oportunidades puede tratarse a partir de la comparación de los resultados económicos condicionados a los conjuntos de variables de oportunidad. Véanse Pattanaik y Xu (1990) y Kranich (1996) para obtener información más detallada. En Ooghe, Schokkaert y Van de Gaer (2007) se ofrece un resumen de los distintos enfoques.

${ }^{3}$ Véanse, por ejemplo, Dworkin (1981) y Arneson (1989).

${ }^{4}$ La discusión relacionada con la norma social de justicia es compleja e implica una serie de visiones que entran en conflicto. Véase una síntesis en Thomson (2011) y Fleurbaey y Maniquet (2011).
} 
No obstante, si bien el concepto de igualdad de oportunidades es bastante simple, su implementación conlleva algunos desafíos significativos, entre los que destaca la definición de la variable de esfuerzo. Fleurbaey (1998) considera que el esfuerzo no es observable y utiliza un enfoque no paramétrico para su identificación, basado en el supuesto de identificación de Roemer según el que supone que las variables de esfuerzo multidimensionales se pueden distribuir independientemente de las circunstancias de los individuos. Por otra parte, en Bourguignon, Ferreira y Menéndez (2007) se estima que el esfuerzo es observable y se desarrolla un modelo paramétrico en el que, endógenamente, el esfuerzo depende de las circunstancias. En resumen, el esfuerzo puede considerarse una variable latente (no observable) $\mathrm{o}$ no ${ }^{5}$. Al adoptar una estructura en que este es un factor observable, las representaciones paramétricas tradicionales parten de la base de que el resultado económico, como por ejemplo el salario, es una función de las variables de circunstancias $\left(C_{i}\right)$ y de esfuerzo $\left(E_{i}\right)$ siguiendo una estructura aditivamente separable:

$$
Y_{i}=\alpha C_{i}+\lambda E_{i}+u_{i}
$$

La ecuación (1), sin embargo, requiere que se disponga de los dos conjuntos de variables (de esfuerzo y de circunstancias), lo que — como norma general— no es posible en la mayoría de las encuestas internacionales. Ante esto, en diversos estudios se supone que la determinación del resultado económico se puede realizar a partir de una serie de variables de esfuerzo junto con supuestos relativos al término aleatorio.

Devooght (2008), por ejemplo, aplica un criterio normativo en virtud del cual el término de error se incluye en el conjunto de variables de circunstancias $\left(C_{i}\right)$. Siguiendo la misma línea de razonamiento, en Almås (2008) y Almås y otros (2011) se elabora un criterio de justicia sensible a la responsabilidad y se propone un coeficiente de Gini de desigualdad injusta, que se aplica a los datos de Noruega. En Figueiredo y Netto Júnior (2014) se utiliza una estrategia similar para calcular las desigualdades injustas en el Brasil entre 1995 y 2009.

\footnotetext{
5 En Bourguignon, Ferreira y Menéndez (2007), por ejemplo, la aproximación en el caso de las variables de esfuerzo se realiza a partir de los años de estudio, la decisión de migrar y la situación del trabajador en el mercado laboral. Checchi y Peragine (2009) consideran el esfuerzo un factor no observable, con lo que asumen el axioma de identificación de Roemer (1996) y suponen que individuos con distintos conjuntos de oportunidades, pero que están en el mismo percentil de la distribución condicionada a su tipo, realizan el mismo nivel de esfuerzo.
}

Los resultados relacionados con la economía brasileña resultan llamativos, ya que el país presenta una significativa reducción de la desigualdad total de los ingresos (coeficiente de Gini), sin una alteración de la desigualdad injusta. Dado que el cálculo de los coeficientes de desigualdad injusta se basa en variables de esfuerzo (educación, horas de trabajo, decisión de migrar y situación en el mercado laboral), si se mantienen los coeficientes significa que el peso de esas variables covariantes en la explicación de las ecuaciones de ingresos ha permanecido constante a lo largo de los años. De hecho, las ecuaciones presentes en Figueiredo y Netto Júnior (2014) demuestran que se ha producido una caída en el grado de ajuste de las regresiones $\left(R^{2}\right)$. Ante esa constatación, los autores postulan lo siguiente: i) dado que en la construcción del criterio de justicia se considera que los factores no observables (término de error) son las variables ajenas a la responsabilidad, la disminución de $R^{2}$ supone que esos factores tienen mayor peso en 2009 que en 1995. En otras palabras, la densidad de ingresos en 2009 depende mucho más de variables relacionadas con el origen, el color de la piel y los antecedentes familiares que de factores relacionados con el esfuerzo; o bien

ii) un patrón de este tipo se produciría, por ejemplo, si con la expansión cuantitativa del acceso a la educación, las diferencias en la calidad de la educación tendieran a resultar más importantes con el tiempo (Figueiredo y Netto Júnior, 2014).

No obstante, es necesario plantear al menos una reserva: los autores utilizan una estructura paramétrica lineal similar a la de la ecuación (1). En caso, por ejemplo, de que esa especificación no sea apropiada y la relación entre los ingresos y las variables de esfuerzo presenten una estructura no lineal, los valores previstos según las ecuaciones estimadas (utilizados para la elaboración de los coeficientes de desigualdad injusta) y, por consiguiente, los grados de ajuste de las regresiones $\left(R^{2}\right)$ no serán válidos ${ }^{6}$.

Ante esa limitación, en este estudio se propone un modelo alternativo que representa así el resultado económico:

$$
\ln Y_{i}=m\left(E_{i}\right)+g\left(\xi_{i}\right)
$$

donde $g\left(\xi_{i}\right)=s\left(C_{i}, u_{i}\right)$. Se observa también que, al contrario de lo que sucede en la ecuación (1), la

\footnotetext{
6 Véase en Maasoumi, Racine y Stengos (2007) una discusión sobre el grado de ajuste en modelos no lineales y una presentación de alternativas a ese parámetro.
} 
ecuación (2) no impone una forma lineal para describir la vinculación entre el resultado económico y sus variables covariantes. En lugar de eso, se supone que esta relación se da a partir de funciones desconocidas, $m(\cdot), g(\cdot) \mathrm{y}$ $n(\cdot)$. Por lo demás, se considera que $E_{i} \perp \xi_{i}$, es decir, se postula la independencia entre los dos conjuntos de variables ${ }^{7}$. En resumen, se conjugan los enfoques inspirados en la suposición normativa de Devooght (2008) con la estructura no paramétrica ampliamente adoptada en estudios económicos ${ }^{8}$. La aplicación de esos métodos sigue el cronograma empírico que se describe a continuación.

\section{a) Cronograma de la ejecución empírica}

La estrategia empírica incluye los siguientes procedimientos:

1) Realización de análisis de especificación con el fin de comparar las alternativas paramétrica y no paramétrica para las ecuaciones de ingresos. Esta etapa es necesaria a objeto de evitar que se adopte de manera ad hoc la estructura no paramétrica.

2) Creación de grupos de alto y bajo esfuerzo. Se consideran cuatro divisiones, cada una de ellas vinculada a una dimensión del esfuerzo, a saber:

- Educación: bajo esfuerzo en el caso de personas con menos de cuatro años de estudio y alto esfuerzo en los demás casos.

- Migración: bajo esfuerzo para los no migrantes y alto esfuerzo para los migrantes.

- Horas de trabajo: bajo esfuerzo para las personas que trabajan menos de 20 horas semanales y alto esfuerzo para las demás.

- Situación en el mercado laboral: bajo esfuerzo para los trabajadores por cuenta propia e informales y alto esfuerzo para los trabajadores del sector formal.

La idea consiste en medir la distancia entre los grupos de bajo y alto esfuerzo en un período que comprende varios años. La identificación se basa en los valores ajustados de las regresiones. De esa forma, es posible averiguar si el esfuerzo va perdiendo su influencia en la determinación de los ingresos de los brasileños.

3) Utilizando los valores ajustados de las regresiones no paramétricas, se elaboran criterios de justicia y coeficientes de Gini de desigualdad injusta.

4) Este procedimiento permite establecer si la ganancia obtenida en la aplicación de un método

\footnotetext{
${ }^{7}$ Esta hipótesis se relaja en la sección dedicada a los análisis de robustez.

8 Véase una síntesis en Li y Racine (2007).
}

no paramétrico para las regresiones de salarios se refleja significativamente en los índices de injusticia.

Por último, se realiza un análisis de robustez basado en una muestra con variables de esfuerzo y de circunstancias.

Los ejes del análisis son la hipótesis de la independencia entre los dos conjuntos de variables y la omisión de las variables circunstanciales en los procedimientos 1) a 3). Se utilizan datos del suplemento social de la PNAD de 1996.

La implementación de este cronograma (procedimientos 1 a 4) se basa en un instrumental no paramétrico y en un conjunto de datos que se presentan en las dos subsecciones siguientes.

\section{b) Inferencia}

Se utilizan tres métodos no paramétricos. El primero es un análisis de especificaciones y se relaciona con el procedimiento 1) de la estrategia empírica, es decir, se comprueba la robustez de la especificación no paramétrica en comparación con la paramétrica. El segundo se refiere a las estimaciones no paramétricas para las ecuaciones salariales. El tercero, finalmente, hace referencia al cálculo de las distancias (entropías) entre los grupos de bajo y alto esfuerzo.

Análisis de las especificaciones. En este estudio se opta por el análisis basado en núcleos (kernel) desarrollado por Racine (2006). Para entender la estructura del análisis de especificación de Racine (2006) hay que considerar la representación paramétrica:

$$
y_{i}=q\left(x_{i}, \beta\right)+\eta_{i}, i=1, \ldots, n
$$

En caso de que la especificación sea correcta, la esperanza del error $\eta_{i}$ condicionada a $x_{i}$ será igual a cero. De esta forma, el estimador para $\beta$ será consistente. En cambio, en caso de que $q\left(x_{i}, \beta\right)$ estuviera mal especificada, siguiendo la estructura habitual de regresión lineal, por ejemplo, el estimador de $\beta$ sería inconsistente. En consecuencia, habría que considerar la especificación correcta como la hipótesis nula9:

$$
H_{0}: P\left[F\left(q\left(x_{i}, \beta\right) \mid x_{i}\right)\right]=1 \text {, para algún } \beta \in \aleph \forall i \geq 1
$$

\footnotetext{
9 La hipótesis nula se basa en la definición de análisis consistente. Para mayor información, véase Li y Racine (2007).
} 
donde $q\left(x_{i}, \beta\right)$ es una función conocida, $\beta$ es un vector de parámetros desconocidos y $\aleph$ es un subconjunto compacto de $\mathbb{R}^{d}$ la hipótesis alternativa es la siguiente:

$$
H_{1}: P\left[F\left(q\left(x_{i}, \beta\right) \mid x_{i}\right)\right]<1 \text {, para algún } \beta \in \aleph \forall i \geq 1
$$

La hipótesis nula es verdadera si $E\left(\varepsilon_{i} \mid x_{i}\right)=0$, siendo $I=E\left\{\left[E\left(\varepsilon_{i} \mid x_{i}\right)\right]^{2} f\left(x_{i}\right)\right\} \geq 0$, donde $f\left(x_{i}\right)$ es la densidad marginal para $X$. En este caso, $I=0$ si y solamente si $H_{0}$ es verdadera. Así pues, $I$ es un candidato para analizar $H_{0}$.

En este punto conviene realizar una observación. Por regla general, los métodos basados en núcleos son apropiados para datos continuos. Sin embargo, empleando un método de frecuencias (Li y Racine, 2007, cap. 3), es posible tratar, en un mismo conjunto de datos, variables discretas y continuas. De este modo, se puede considerar $x_{i}^{d}$ como un vector de variables discretas de dimensión $r \times 1$ y $x_{i}^{c} \in \mathbb{R}^{d}$ como el resto de las variables continuas. De esta forma, $x_{i}=\left(x_{i}^{c}, x_{i}^{d}\right)$.

El I muestral se define como:

$$
I_{n}=n^{-2} \sum_{i} \sum_{j \neq i} \hat{\varepsilon}_{i} \hat{\varepsilon}_{j} K_{\gamma, i j}
$$

donde $K_{\gamma, i j}=W_{h, i j} L_{\lambda, i j}, \gamma=h, \varphi$ son los anchos de banda, $W_{h, i j}$ y $L_{\lambda, i j}$ son las funciones kernel multivariantes para los datos discretos y continuos, respectivamente, y $\hat{\varepsilon}_{i}$ son los errores muestrales del modelo.

En Racine (2006) se recomienda la utilización del método de validación cruzada para la selección de los anchos de banda ${ }^{10}$, sustituyendo $\left(h_{1}, \ldots, h_{q}, \varphi_{1}, \ldots, \varphi_{r}\right)$ por sus estimaciones $\left(\hat{h}_{1}, \ldots, \hat{h}_{q}, \hat{\varphi}_{1}, \ldots, \hat{\varphi}_{r}\right)$. De esta manera, se define $\hat{I}_{n}$. En algunos supuestos, el autor demuestra que, con $H_{0}$,

$$
\hat{J}_{n}=n\left(\hat{h}_{1}, \ldots, \hat{h}_{q}\right)^{1 / 2} \hat{I}_{n} / \sqrt{\hat{\Omega}} \rightarrow N(0,1)
$$

con

$$
\hat{\Omega}=\frac{2\left(\hat{h}_{1}, \ldots, \hat{h}_{q}\right)}{n^{2}} \sum_{i} \sum_{j \neq i} \hat{\varepsilon}_{i}^{2} \hat{\varepsilon}_{j}^{2} W_{\hat{h}, i j}^{2} L_{\hat{\lambda}, i j}^{2}
$$

10 Véase Härdle (1990) para obtener más información sobre el método de validación cruzada.
Los valores críticos se tabulan según el método de wild bootstrap. En Racine (2006) se presentan y discuten detalles relativos al proceso de inferencia, así como el desempeño del análisis en muestras finitas. A grandes rasgos, el análisis $\hat{J}_{n}$ consta de diversas etapas, entre las que destacan la estimación de regresiones con datos combinados, o mixed data (Racine y Li, 2004), con la obtención de estimaciones para los anchos de banda de variables discretas y continuas.

Estimaciones no paramétricas. En muchos de los estudios empíricos se admite una forma funcional específica, en general log-lineal, para la medición de la desigualdad de oportunidades. Cabe citar los de Bourguignon, Ferreira y Menéndez (2007); Devooght (2008), y Figueiredo y Netto Júnior (2014), entre otros. Sin embargo, es posible que la modelización paramétrica no sea apropiada, teniendo en cuenta la incertidumbre referente a las relaciones entre la variable explicada y las explicativas. Alternativamente, una estimación no paramétrica se caracteriza por no imponer formas funcionales para la ecuación, de modo que es capaz de revelar aspectos de la estructura de los datos no captados por el enfoque paramétrico tradicional.

En este estudio se utiliza el estimador kernel local constante para datos mixtos, sugerido por Racine y Li (2004), teniendo en cuenta que las regresiones incluyen variables categóricas. Los autores utilizan una función de kernel para el suavizado de las variables discretas, que adopta las siguientes formas:

$$
l\left(X_{t, i}^{d}, x_{t}^{d}\right)=\left\{\begin{array}{l}
1 \text { se } X_{t, i}^{d}=x_{t}^{d} \\
\lambda \text { se } X_{t, i}^{d} \neq x_{t}^{d}
\end{array}\right.
$$

Se define $d_{x i, x}=\sum_{t=1}^{k} 1\left(X_{t, i}^{d} \neq x_{t}^{d}\right)$ como el número de componentes desiguales entre $X_{t, i}^{d}$ y $x_{t}^{d}$. Así, el kernel de producto (product kernel) para variables discretas será

$$
L\left(X_{i}^{d}, x^{d}, \lambda\right)=\prod_{t=1}^{k} l\left(X_{t, i}^{d}, x_{t}^{d}\right)=1^{k-d_{x i, x}} \lambda^{d_{x i, x}}=\lambda^{d_{x i, x}} .
$$

Sea W(.) la función de kernel asociada a las variables continuas y $h$ sus parámetros de suavizado. Usando la notación $K_{h, i x}=W_{h, i x} L_{\lambda, i x}$, donde $W_{h, i x}=h^{-p} W\left(\left(X_{i}^{c}-x^{c}\right) / h\right)$ y $L_{\lambda, i x}=L\left(X_{i}^{d}, x^{d}, \lambda\right)$, el estimador de kernel para la función de densidad conjunta de $\left(X_{i}^{c}, X_{i}^{d}\right)$ está dado por: 


$$
\hat{f}(x)=\frac{1}{n} \sum_{i=1}^{n} K_{h, i x}
$$

En resumen, la verdadera función de los ingresos corresponde al promedio condicional de la variable dependiente, y su estimador se define como:

$$
\hat{g}(x)=\frac{n^{-1} \sum_{i=1}^{n} Y_{i} K_{h, i x}}{\hat{f}(x)}
$$

donde $Y_{i}$ es la variable dependiente y $K_{h, i x}$ responde a la definición antes formulada. Los resultados de esta función se resumen en forma de gráficos de regresión parcial. Se traza la parte explicada de la regresión multivariante en relación con cada variable de esfuerzo, manteniendo las demás constantes, en una serie de gráficos bivariantes. Es decir, se traza $\hat{g}$ con respecto al valor esperado de los regresores, manteniéndose dos de estos en sus respectivos promedios; todo ello para dos grupos diferentes de individuos de acuerdo con una división entre bajo esfuerzo y alto esfuerzo, según cada factor de responsabilidad analizado. Este procedimiento, utilizado en Li, Maasoumi y Racine (2009), permite comparar las curvas de ambos grupos usando como base la distancia entre ellas y, de esta forma, observar la capacidad de esos regresores de explicar la desigualdad de ingresos a lo largo del tiempo. La cuantificación de esas distancias se realiza a través de una medida de entropía, que se explica con más detalle en el apartado siguiente.

Medida de la entropía de las distancias. Las medidas de entropía representan una cuantificación formal de las distancias entre las distribuciones. Una medida ideal de la distancia entre dos variables aleatorias presenta una serie de propiedades deseables, a saber: i) está normalizada a cero, en caso de que $X$ e $Y$ sean idénticas; ii) el módulo de la medida es igual a uno, en caso de que exista una relación exacta (no lineal) medible entre las variables $Y=g(X)$; iii) tiene un valor próximo al coeficiente de correlación lineal en el caso de una distribución normal bivariante; iv) es una verdadera medida de distancia y no solo de divergencia entre las distribuciones; v) la medida ideal está bien definida tanto para variables continuas como para variables discretas, y vi) es invariante ante transformaciones continuas y estrictamente crecientes como $h($.). Esto resulta útil siempre que $X$ e $Y$ sean independientes y si, y solamente si, $h(\mathrm{X})$ y $h(\mathrm{Y})$ también son independientes.
Li, Maasoumi y Racine (2009) utilizan las medidas de entropía para realizar inferencias sobre el grado de ajuste de los modelos, midiendo la distancia entre valores observados y valores ajustados, ya que el $R^{2}$ tradicional y otras medidas de correlación resultan inadecuados cuando se admite que la función estimada no es lineal. La entropía empleada por los autores fue sugerida por Granger, Maasoumi y Racine (2004) y consiste en una normalización que posee las propiedades anteriormente indicadas, así como una métrica de distancia apropiada:

$$
S_{\rho}=\frac{1}{2} \int\left(f_{1}^{1 / 2}-f_{2}^{1 / 2}\right)^{2} d x
$$

donde $f_{1}$ y $f_{2}$ son las densidades marginales de las variables aleatorias. Para una mayor fiabilidad de los resultados, el método de bootstrap se aplica a través de un nuevo muestreo con reajuste de la distribución conjunta de $X$ e $Y$.

Como se ha mencionado, en este estudio se utilizarán las medidas de entropía como un indicador de la desigualdad de esfuerzo entre los grupos (procedimiento 3). Los valores ajustados de la variable dependiente para los individuos con bajo esfuerzo y con alto esfuerzo se trazarán en relación con cada variable explicativa, manteniéndose constantes las demás. A través de las medidas de entropía se calculan las distancias entre las curvas e inmediatamente se realiza un análisis de igualdad de densidades univariantes, que se basa en la hipótesis nula $S_{\rho}=0$.

\section{c) Datos}

Los datos utilizados en el estudio proceden de la PNAD respecto del período comprendido entre 1995 y 2009. Se consideran los varones jefes de familia, con edades entre 25 y 60 años, residentes en áreas urbanas. La selección de individuos con estas características se realizó con el objetivo de homogeneizar al máximo la muestra utilizada, restringiéndose el análisis a los individuos en edad activa, además de evitarse la influencia de factores de género en la selección y en los resultados económicos individuales. Se eligió a los jefes de familia porque representan la principal fuente de ingresos familiares. Los ingresos están representados por el salario por hora, expresado en reales de septiembre de 2009, como en Bourguignon, Ferreira y Menéndez (2007).

El vector de variables de esfuerzo está representado por: i) años de estudio del individuo; ii) una variable ficticia para la migración; iii) su situación en el mercado laboral (trabajador formal, sin contrato de trabajo o 
que trabaja por cuenta propia), y iv) horas semanales de trabajo. Para cada una de las variables se definieron las categorías de alto esfuerzo y bajo esfuerzo, según se indica en el procedimiento 2 del Cronograma de la ejecución empírica (véase la subsección 1.a de la sección II).

Para la selección de las variables se siguen los estudios de Bourguignon, Ferreira y Menéndez (2007) y Figueiredo y Netto Júnior (2014). Esas variables se consideran factores de responsabilidad de los individuos, dado que, aunque se ven influidas por las circunstancias, en última instancia están determinadas por las opciones individuales. En relación con los años de estudio, es razonable suponer que los individuos con más nivel de escolaridad son los que han intentado obtener una más alta calificación, han invertido en capital humano y, por lo tanto, han realizado un mayor esfuerzo.

\section{III}

\section{Resultados}

En esta sección se muestran los resultados de cada uno de los pasos enumerados en la estrategia empírica. En primer lugar, se procede a los análisis de especificaciones para las ecuaciones de ingresos. A continuación, se realiza un análisis gráfico de las densidades no paramétricas, comparando las diferencias de la renta entre los grupos de alto esfuerzo y de bajo esfuerzo a lo largo del período analizado, haciéndose variar por separado cada factor de responsabilidad. La cuantificación formal de esas distancias se obtiene a partir de las medidas de entropía para las distancias entre las densidades de los dos grupos. Finalmente, se realizan los análisis de robustez, en que se utilizan datos sobre las circunstancias de los individuos, así como la medición y comparación de los resultados relacionados con la desigualdad de oportunidades no paramétrica en el Brasil.

Paso 1. Los análisis de las especificaciones de las ecuaciones de ingresos se resumen en el cuadro 1. Como ya se ha indicado, se trata de comparar las estimaciones
Se consideran las variables de situación en el mercado laboral y horas semanales trabajadas, ya que representan la búsqueda de mejores salarios, puesto que los individuos que trabajan más horas y en empleos formales tienden a obtener ingresos superiores. Además, en general, los empleos formales están ocupados por individuos con un nivel de escolaridad más alto.

En cuanto al factor migratorio, se puede afirmar que los individuos que deciden migrar son seleccionados por el mercado de trabajo y, según Assis, Costa y Mariano (2012), se trata de individuos que presentan un nivel más alto de capital humano, además de ser más productivos que los no migrantes, excepto en la región Sudeste. Teniendo en cuenta esas características, los migrantes tendrían la capacidad de alcanzar un nivel de ingresos superior, lo que hace que la inclusión de esta variable como medida de esfuerzo resulte plausible. paramétrica y no paramétrica para las ecuaciones de ingresos. Con este procedimiento se evita la decisión relativa a la adopción ad hoc de una estructura de estimación. En resumen, el análisis desarrollado por Racine (2006) demuestra la superioridad de los modelos no paramétricos en todos los años investigados.

Paso 2. Una vez demostrada la superioridad de los modelos no paramétricos, el estudio pasa a las inferencias basadas en el estimador kernel constante ${ }^{11}$. Después de la obtención de los valores ajustados para cada ecuación, se identifican los grupos de alto y bajo esfuerzo, según las categorías indicadas en la sección II. En el gráfico 1 se presentan las estimaciones de las densidades kernel para los valores ajustados condicionados a los grupos de esfuerzo en 1995 y 2009.

\footnotetext{
${ }^{11}$ Los resultados de las estimaciones se eliminaron por limitaciones de espacio. Pueden solicitarse por correo electrónico al autor correspondiente.
} CUADRO 1

Análisis de especificaciones paramétricas

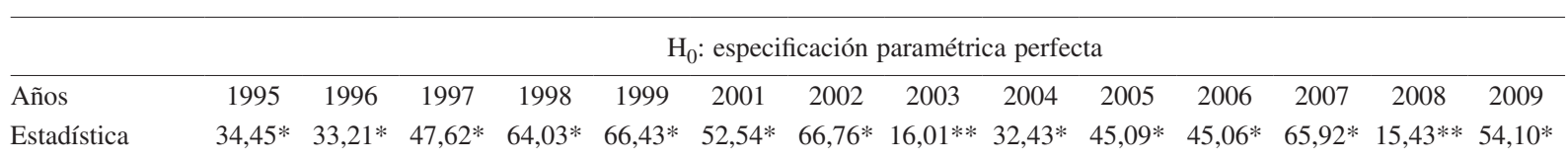

Fuente: elaboración propia.

Nota: * y ** representan el rechazo de la hipótesis nula con un $1 \%$ y un 5\% de significación, respectivamente. 
GRÁFICO 1

Brasil: densidades no paramétricas para los valores ajustados por factores de esfuerzo, 1995 y 2009

A. Educación, 1995

Distribución de los valores ajustados

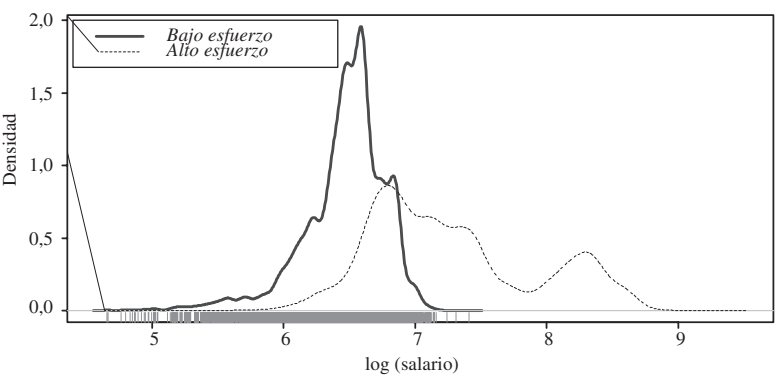

C. Migración, 1995

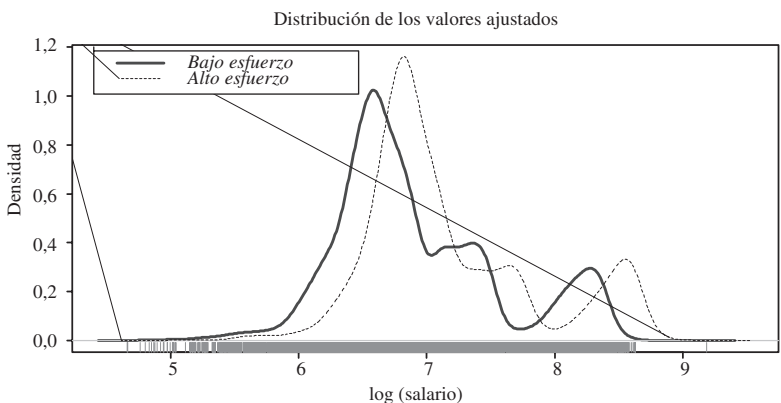

E. Situación en el mercado laboral, 1995

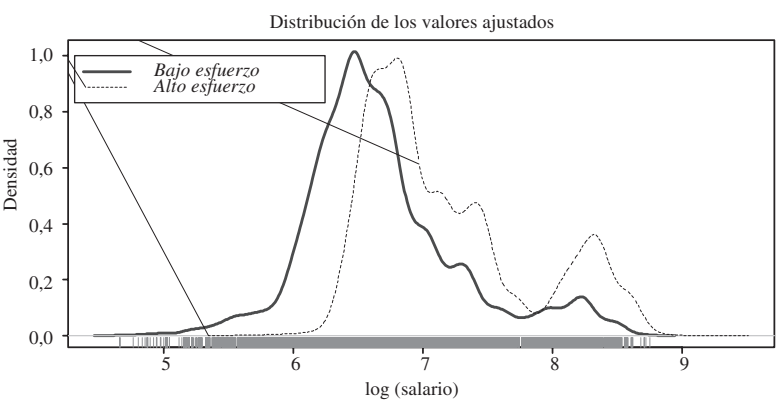

G. Horas trabajadas, 1995

Distribución de los valores ajustados

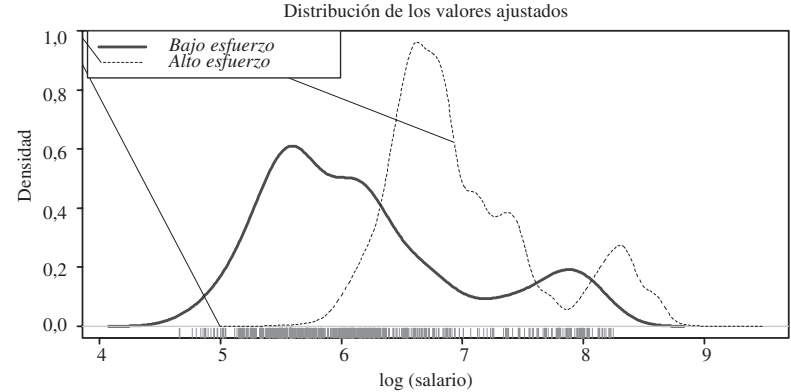

B. Educación, 2009

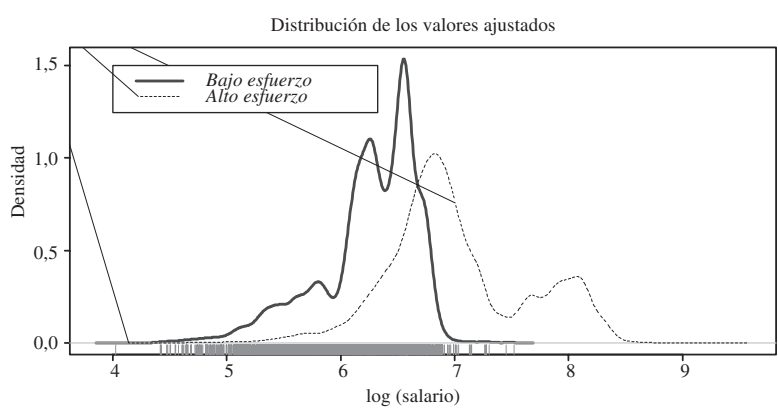

D. Migración, 2009

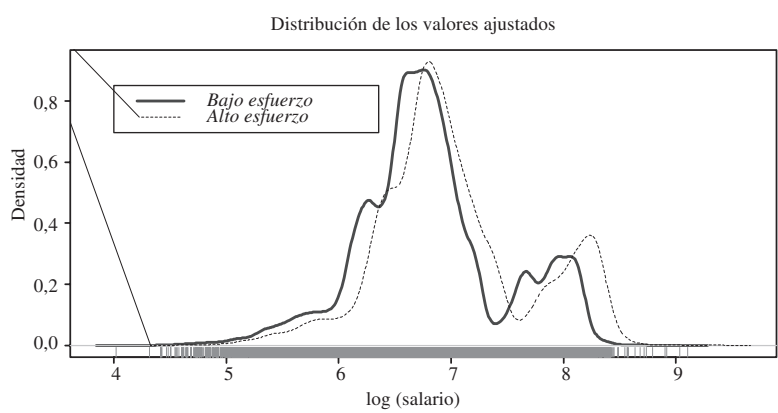

F. Situación en el mercado laboral, 2009

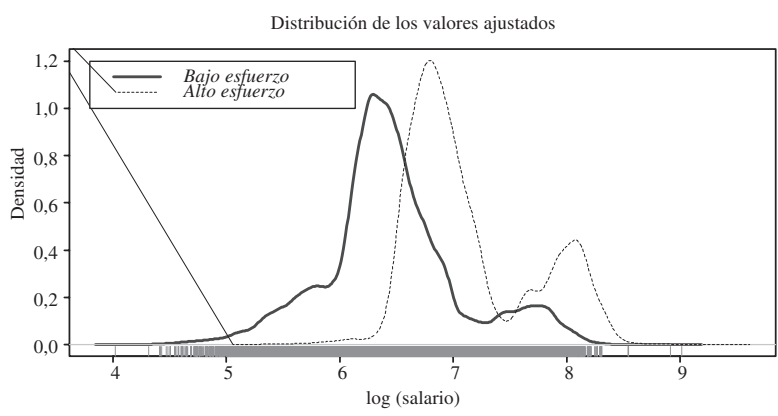

H. Horas trabajadas, 2009

Distribución de los valores ajustados

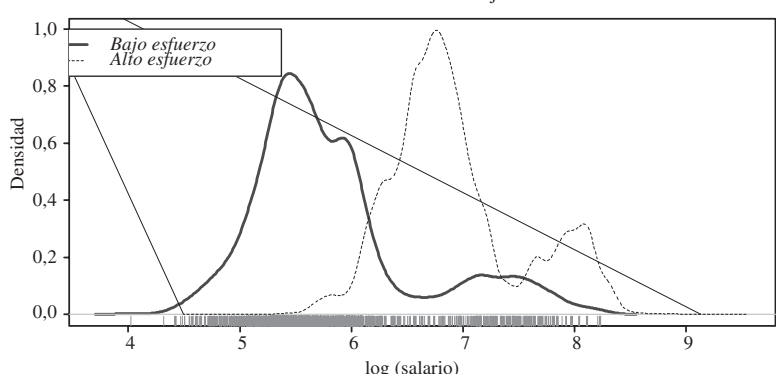

Fuente: elaboración propia. 
Un análisis visual indica que se encontró una aproximación entre las curvas de la educación como criterio de desigualdad, además de una menor dispersión en la distribución de los individuos de la categoría de alto esfuerzo. Con respecto a la migración, se puede percibir que la diferencia entre los grupos, tanto en 1995 como en 2009, es poco significativa y apenas varía de un año a otro.

Al considerarse la situación en el mercado laboral, se observa un aumento en la distancia entre las densidades, con una leve reducción de la dispersión de la curva correspondiente al grupo de bajo esfuerzo. De forma análoga, la diferencia entre las distribuciones atribuida al factor de horas trabajadas se acrecentó en el período, sin grandes alteraciones en la forma de las curvas.

No obstante, es necesario realizar una cuantificación formal de las distancias entre las curvas sintetizadas en el gráfico 1, además de mostrar su evolución durante el período analizado. En el gráfico 2 se representa la evolución de las distancias entre los valores ajustados de los grupos de alto y bajo esfuerzo, para cada dimensión del esfuerzo, a lo largo del período considerado ${ }^{12}$. Cualquier medida de entropía es útil como indicador de divergencia entre distribuciones y constituye, por lo tanto, una medida de la desigualdad o concentración de estas.

Al considerar la educación como factor de esfuerzo, los valores de las entropías disminuyen durante el período

${ }^{12}$ Los valores de las entropías para cada año de la serie se muestran en el cuadro A.1 del anexo de este artículo. en conjunto. De 1995 a 2009, la distancia entre los grupos, que había sido de poco más de 0,50 en 1997, cayó a un valor cercano a 0,36 en 2009, lo que indica que el esfuerzo a través del aumento de nivel educacional ha ido perdiendo importancia en la determinación de las diferencias de ingresos entre los individuos. Es decir, los años de estudio en 1995 (entropía = 0,41) suponían un nivel de ingresos mayor que en 2009 (entropía = 0,35 ) con respecto a los individuos que tenían un nivel educativo inferior.

En cuanto a la migración, los valores de las entropías son inferiores a los de las demás variables de esfuerzo, lo que sugiere que esta variable tiene un bajo poder explicativo. Así, el hecho de que un individuo sea migrante no se traduce en una mejora significativa de sus ingresos.

Se puede encontrar una razón de esa escasa repercusión de la migración en el estudio de Assis, Costa y Mariano (2012), en que se utilizan datos de migrantes no naturales, migrantes de retorno y no migrantes de los estados de São Paulo y Bahia, que sirven como referencia para las regiones Sudeste y Nordeste, respectivamente. Sus resultados indican que los migrantes de la región Nordeste, sobre todo los de retorno, tienen un nivel de ingresos superior a los no migrantes, mientras que en São Paulo se observa lo contrario, ya que los migrantes de este estado son individuos que no han conseguido espacio en un mercado local más dinámico. Por lo tanto, dado que para este estudio se utilizaron datos referentes al Brasil, esos efectos pueden haberse anulado mutuamente, de tal modo que, en conjunto, la migración no haya

GRÁFICO 2

Evolución de las entropías, 1995-2009

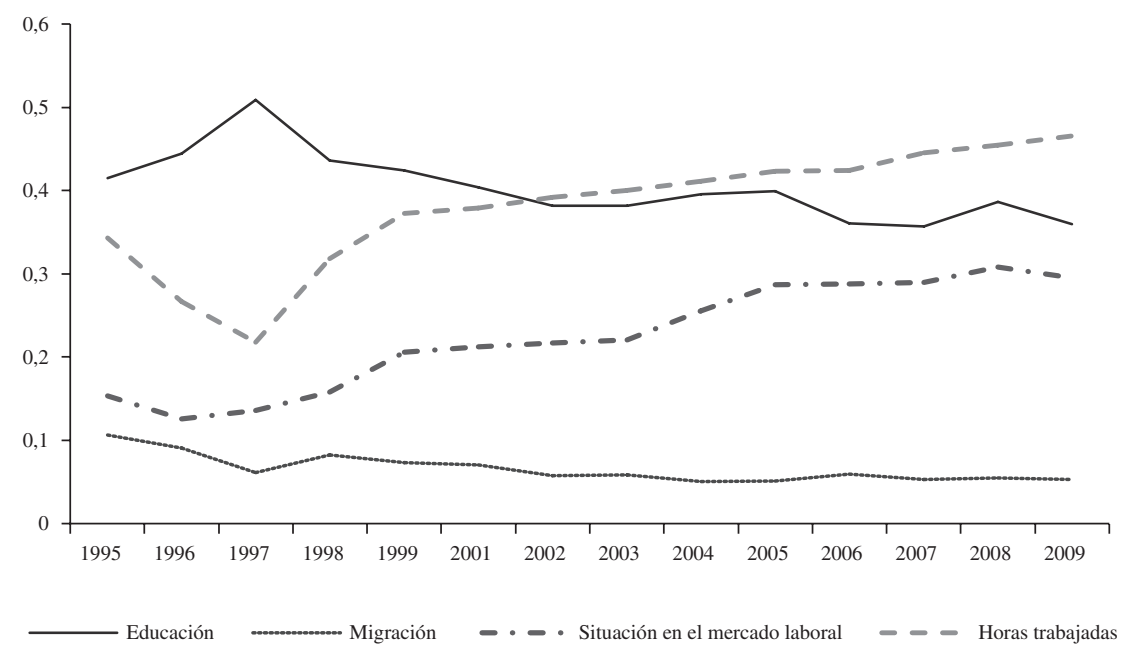

Fuente: elaboración propia. 
representado una diferencia de salarios significativa entre los grupos.

Por otra parte, durante el período aumentaron las distancias $\left(S_{p}\right)$ para las variables de situación en el mercado laboral y horas semanales trabajadas. Los valores de las entropías para las horas trabajadas fueron en todos los años mayores que los valores asociados a la situación en el mercado laboral, lo que muestra la mayor relevancia de la primera variable en comparación con la segunda en la explicación de la desigualdad de ingresos entre los grupos. La evolución de las entropías con el paso del tiempo refleja una considerable variación de las distancias para ambas variables con respecto al año inicial.

Paso 3. La pregunta principal en esta etapa es qué impacto tiene la utilización de inferencias no paramétricas en los indicadores de justicia. Figueiredo y Netto Júnior (2014) proponen una estimación de los índices de desigualdad injusta en el período comprendido entre 1995 y 2009. Los autores emplean, básicamente, modelos paramétricos, aunque se aborden cuestiones relacionadas con la endogeneidad de las ecuaciones y la identificación de los parámetros. No es objeto de este estudio proponer estimaciones más robustas para las medidas de desigualdad injusta, dado que las inferencias puntuales, sean paramétricas o no, pueden presentar sesgos.

Por lo tanto, solo se presenta la comparación de los índices de desigualdad injusta (véase el gráfico 3 ) calculados sobre la base de una especificación paramétrica con estos mismos indicadores calculados a partir del método no paramétrico ${ }^{13}$.

Cabe destacar que, pese a que el comportamiento es similar, el índice de desigualdad injusta calculado a partir de la especificación (2) presenta siempre un valor inferior al calculado por mínimos cuadrados ordinarios (MCO). En resumen, hay una diferencia promedio del 10\% entre estas inferencias, de modo que el método paramétrico tiende a sobrestimar el índice de desigualdad injusta.

Paso 4. Los resultados presentados hasta el momento dependen de una hipótesis fundamental: la independencia entre las variables de responsabilidad y de circunstancias. Es decir, se postula que las horas de trabajo, la educación, la decisión de migrar y la situación en el mercado laboral no se ven afectadas por las variables de circunstancia, como los antecedentes familiares, el género o la raza.

Se recurre con frecuencia a esa suposición en la bibliografía sobre la desigualdad de oportunidades (véanse O'Neill, Sweetman, D. y Van de Gaer, 2001, y Checchi y Peragine, 2009). Sin embargo, desarrollos recientes de algunas teorías sobre justicia alertan sobre la posibilidad de que los factores de responsabilidad y los ajenos a la responsabilidad no sean independientes (Betts y Roemer, 2005). El estudio de Bourguignon, Ferreira y Menéndez (2007) constituye una destacada referencia

13 Véanse Almås (2008) y Almås y otros (2011) para obtener información sobre el desarrollo del criterio de justicia y el índice de desigualdad injusta.

GRÁFICO 3

Brasil: índices de desigualdad injusta

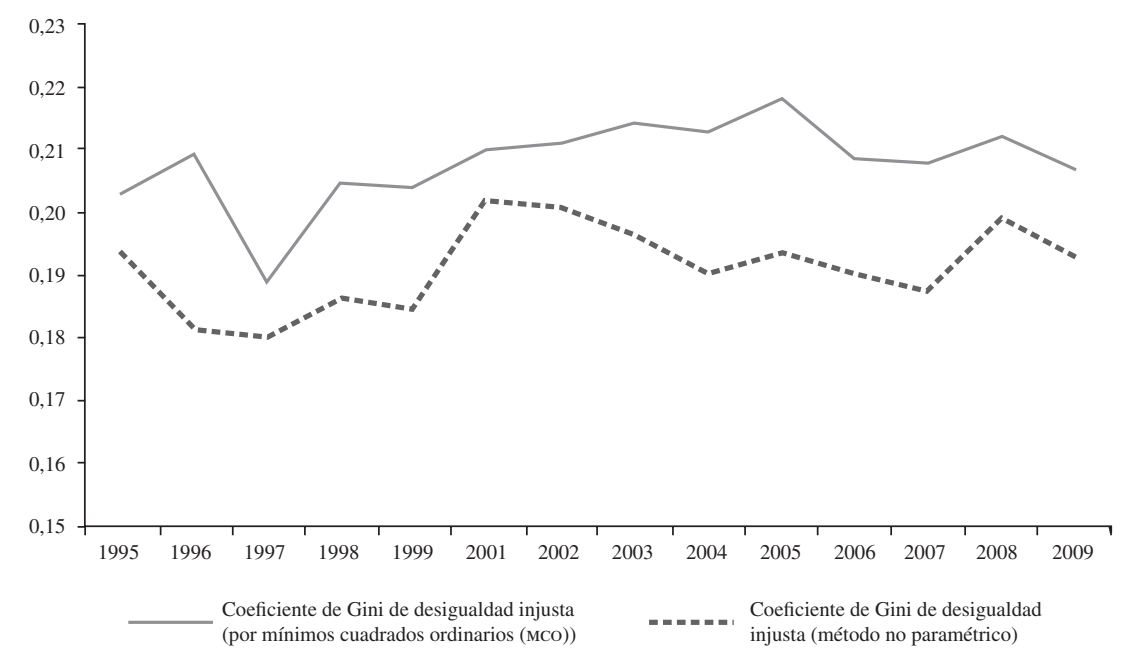

Fuente: elaboración propia. 
empírica para esa hipótesis. Con datos sobre el Brasil, estos autores demuestran que las circunstancias tienen efectos directos e indirectos en la desigualdad total. El efecto indirecto refleja la influencia de las circunstancias en el esfuerzo. En resumen, las circunstancias son responsables de cerca del $23 \%$ de la desigualdad total: un $13 \%$ por efecto directo y un $10 \%$ por efecto indirecto.

Así, es posible que en los resultados obtenidos hasta entonces influyera la hipótesis de independencia entre los dos conjuntos de variables. Por lo tanto, se precisa un análisis de robustez basado en un procedimiento simple: debe aislarse el efecto de las circunstancias en el esfuerzo, en particular en los años de estudio, y analizarse si la omisión de las variables de circunstancias afecta a las entropías ${ }^{14}$.

Para los análisis de robustez, se estima una ecuación en que los años de estudio se expliquen mediante un conjunto de variables ajenas a la responsabilidad. Se obtiene así el total de años de estudio sin el efecto de las circunstancias (años de estudio menos años de estudio previstos por las variables); se calculan las distancias de las entropías entre los grupos de bajo y alto esfuerzo, considerando esta nueva variable $\mathrm{y}$, por último, se comprueba si la distancia entre los dos grupos es significativa.

Los datos utilizados en las estimaciones provienen del suplemento social de la PNAD para 1996, donde

${ }^{14}$ Este procedimiento también se empleó para las restantes variables de esfuerzo. Los resultados fueron similares a los de la variable de años de estudio y se eliminaron por falta de disponibilidad de espacio. se resumen las informaciones relacionadas con las circunstancias de los individuos, tales como la educación, la escolaridad y la ocupación de los padres de las personas seleccionadas (antecedentes familiares). De ese modo, para estos años se usan las variables de esfuerzo descritas anteriormente y, además, un vector de variables de circunstancias, definido por: i) una variable ficticia de raza, que adopta el valor 1 en el caso de las personas no blancas (es decir, negras, mulatas e indígenas) y 0 para las personas blancas (blancas y asiáticas); ii) una variable ficticia para las regiones, que adopta el valor 1 si el individuo reside en las regiones más dinámicas del país (Sur, Sudeste y Centro-Oeste) y 0 en caso contrario (regiones Norte y Nordeste); iii) educación del padre y de la madre, expresada en años de estudio, y iv) el tipo de ocupación del padre, según las seis categorías propuestas por Pastore y Silva $(2000)^{15}$.

En el gráfico 4 se muestra la diferencia entre los años de estudio y los años de estudio sin tener en cuenta el efecto de las circunstancias (años de estudio previstos). Hay que destacar que el cálculo de la entropía se realiza a partir de la creación de los grupos de bajo y alto esfuerzo en la variable "años de estudio previstos". Se consideró bajo esfuerzo para los individuos con menos

\footnotetext{
15 Categorías: 1) Nivel bajo inferior: labrador, pescador o leñador, entre otros; 2) Nivel bajo superior: obrero, empleado doméstico o vigilante, entre otros; 3) Nivel medio inferior: albañil, electricista o carpintero, entre otros; 4) Nivel medio medio: vendedor ambulante, viajante o gerente de primer nivel, entre otros; 5) Nivel medio superior: asesor, gerente de alto nivel o director, entre otros, y 6) Nivel alto: gran proprietario, magistrado u otra ocupación de nivel superior.
}

GRÁFICO 4

Brasil: diferencia entre los años de estudio y los años de estudio previstos, 1996

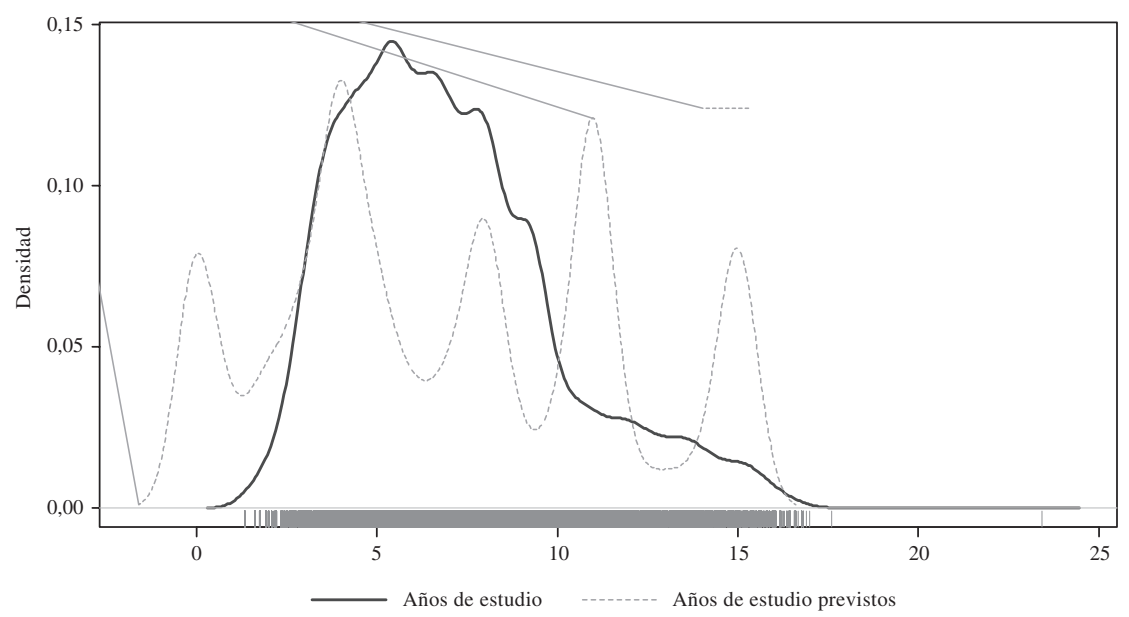

Fuente: elaboración propia. 
de 5 años de estudio previstos (contemplando las dos primeras modas de la curva de trazo discontinuo) y alto esfuerzo para los demás. El resultado para la entropía, de 0,4023 , indica que no se ha producido una reducción significativa, si se compara con la entropía para el año 1996, sin considerar las variables de circunstancias (entropía =0,4447). En ese sentido, aun teniendo en cuenta de que no exista independencia entre los dos conjuntos de variables, de esfuerzo y de circunstancias, no se observa una alteración destacable en la distancia entre los dos grupos de esfuerzo, lo que demuestra la plausibilidad de los resultados presentados en los pasos 1 a 3 .

\section{IV}

\section{Discusión de los resultados}

De acuerdo con lo ya señalado, las medidas de entropía representan una medida de desigualdad de esfuerzo entre los individuos. Al aislarse el efecto de cada variable, se intenta comprobar su poder explicativo acerca de las diferencias de ingresos entre los grupos, cuya cuantificación viene dada por el valor de la distancia entre las distribuciones.

Algunos de los resultados obtenidos deben destacarse por sus implicancias teóricas y prácticas; una de ellas es la reducción de la desigualdad cuando se considera la educación como un factor de esfuerzo. El papel de la educación en la distribución de los ingresos puede contribuir a que se amplíen o se reduzcan las brechas entre los individuos, dependiendo de su grado de retorno y de la composición de la población por nivel educativo. Ferreira y otros (2006), al describir la estructura de la desigualdad de los ingresos en el Brasil, sugieren que la reducción de la desigualdad total correspondiente a las diferencias educativas refleja la expansión de la educación de la fuerza laboral. Probablemente, el aumento de la oferta relativa de personas con enseñanza primaria y media provocó un descenso de la tasa media de retorno de la educación, con lo que se redujo la diferencia salarial entre esas personas y las de baja calificación (Menezes-Filho, 2001).

Otro factor que puede explicar ese resultado es la expansión de programas sociales de transferencias $\mathrm{y}$ el hecho de que se hayan centrado en las personas más pobres. Dado que muchos de los individuos de ese segmento de la población tienen pocos años de escolaridad, los ingresos de las personas del grupo de bajo esfuerzo se elevaron durante el período, por lo que la distancia con el grupo de mayor esfuerzo (mayor nivel de estudios) disminuyó. Otro posible motivo de la reducción de la distancia entre las curvas puede relacionarse con el descenso de la calidad de la educación. Aunque las políticas educacionales han permitido ampliar el promedio de años de estudio de la población, ello no garantiza necesariamente una mayor calificación ni, por lo tanto, tampoco aumentos salariales significativos.

La evolución de la situación en el mercado laboral revela un aumento de las diferencias de ingresos entre trabajadores formales e informales. En general, se cree que el hecho de que el sector formal ofrezca mayores salarios se debe a sus características intrínsecas. Sin embargo, estudios como los de Menezes-Filho, Mendes y De Almeida (2004) muestran claras evidencias de que existe un sesgo de autoselección, lo que indica que la razón por la que el sector formal ofrece mejores salarios se vincula sobre todo a atributos individuales no observables de los propios trabajadores.

Los resultados obtenidos también pueden analizarse a la luz de algunas políticas gubernamentales. Entre estas destacan las que han promovido los programas sociales de transferencias, como Bolsa Família, y los mecanismos de valorización del salario mínimo, usados como instrumentos para reducir las disparidades de ingresos y la pobreza. No obstante, pese a la disminución de la desigualdad en los últimos años en el Brasil, en algunos estudios se cuestiona la influencia efectiva que puedan haber tenido en esa mejora los incrementos del salario mínimo por parte del gobierno (Barros, Carvalho y Franco, 2006) y la contribución del programa Bolsa Família (Soares, 2006).

Con respecto al salario mínimo, desde la implementación del Plan Real se registraron aumentos sucesivos prácticamente todos los años. Sin embargo, estas alzas reales han resultado ineficaces desde el punto de vista de la redistribución, dado que la mayoría de las personas que reciben un salario mínimo no son consideradas pobres según la definición del criterio de pobreza en el Brasil (Saboia, 2006). Un ejemplo de esta situación se da en la región Nordeste, donde una 
parte significativa de los trabajadores perciben ingresos inferiores al salario mínimo y, por lo tanto, no se benefician de los aumentos de este (Instituto de Investigación Económica Aplicada (IPEA). Según Giambiagi y Franco (2007), en el mismo período se redujo el ingreso medio real de los trabajadores.

Entre 1995 y 2009 se registró casi anualmente un aumento salarial superior al de la productividad (incremento del PIB per cápita). En 1997, el ajuste del salario mínimo fue de un 7\%, mientras que la productividad creció en torno del 3\%. Ese mismo año se observó el valor de entropía mínimo con respecto a las horas trabajadas como factor de esfuerzo. Eso confirma la idea de que, dadas las circunstancias, son pocos los incentivos para aumentar la calificación y mejorar la situación en el mercado laboral.

No obstante, la política del salario mínimo afecta significativamente al mercado laboral, pero sobre todo a los trabajadores del sector informal de la economía (Menezes-Filho, Rodrigues y De Souza, 2009). En suma, los ajustes del salario mínimo pueden contribuir a la reducción de la pobreza, si bien es posible observar efectos negativos en el mercado laboral cuando los ajustes son superiores a los aumentos de productividad de los asalariados. Maloney (2000) afirma que el salario mínimo es también un determinante importante del empleo y de los salarios en el sector informal de la mayoría de las economías latinoamericanas.

\section{V}

\section{Consideraciones finales}

En este artículo se ha analizado la evolución de la desigualdad de oportunidades en el Brasil entre 1995 y 2009, condicionada a un conjunto de variables de esfuerzo. Para tal efecto se han utilizado datos de las encuestas nacionales de hogares (PNAD) del período, siguiendo un enfoque no paramétrico presentado en Racine y Li (2004), tanto para la estimación de la función de los ingresos como para la determinación de la distribución de estos entre los individuos. Se crearon dos grupos, uno de bajo esfuerzo y otro de alto esfuerzo, y se evaluó la desigualdad entre ambos empleando medidas de entropía de la distancia entre sus niveles de ingresos.

Los resultados muestran una reducción del poder explicativo de los años de estudio en la diferencia de ingresos entre los grupos, así como una participación poco significativa de la migración. El análisis de robustez para 1996, realizado sobre la base de informaciones del suplemento social de la PNAD de ese año, reveló que la omisión para los otros años de las variables de circunstancias, tales como los antecedentes familiares, no alteró significativamente los resultados, lo que corrobora la plausibilidad de la estrategia utilizada. Algunos avances para mejorar el presente estudio serían: la exploración de los verdaderos motivos de la pérdida de poder explicativo de la variable de la educación en la desigualdad de los ingresos; la exploración de políticas óptimas para la reducción de la desigualdad de oportunidades y el estímulo de las diferencias a través del esfuerzo, así como la profundización de las discusiones relativas a los criterios de justicia aplicados en el caso del Brasil. 
ANEXO

CUADRO A.1

Entropías

Factores de esfuerzo

\begin{tabular}{|c|c|c|c|c|}
\hline \multirow[b]{2}{*}{ Años } & \\
\hline & Educación & Migración & $\begin{array}{l}\text { Situación en el } \\
\text { mercado laboral }\end{array}$ & Horas trabajadas \\
\hline 1995 & $0,4151 *$ & $0,1061 *$ & $0,1535^{*}$ & $0,3428^{*}$ \\
\hline 1996 & $0,4447 *$ & $0,0905^{*}$ & $0,1253 *$ & $0,2664^{*}$ \\
\hline 1997 & $0,5094 *$ & $0,0616^{*}$ & $0,1361 *$ & $0,2175^{*}$ \\
\hline 1998 & $0,4358 *$ & $0,0825^{*}$ & $0,1580 *$ & $0,3179 *$ \\
\hline 1999 & $0,4240 *$ & $0,0736^{*}$ & $0,2055 *$ & $0,3726^{*}$ \\
\hline 2001 & $0,4039 *$ & $0,0701 *$ & $0,2122 *$ & $0,3795^{*}$ \\
\hline 2002 & $0,3816^{*}$ & $0,0580 *$ & $0,2171 *$ & $0,3916^{*}$ \\
\hline 2003 & $0,3814 *$ & $0,0588 *$ & $0,2210 *$ & $0,3999 *$ \\
\hline 2004 & $0,3954 *$ & $0,0505^{*}$ & $0,2554^{*}$ & $0,4109^{*}$ \\
\hline 2005 & $0,3994 *$ & $0,0510 *$ & $0,2874 *$ & $0,4234 *$ \\
\hline 2006 & $0,3602 *$ & $0,0596^{*}$ & $0,2880^{*}$ & $0,4246^{*}$ \\
\hline 2007 & $0,3567 *$ & $0,0534 *$ & $0,2898 *$ & $0,4456^{*}$ \\
\hline 2008 & $0,3865^{*}$ & $0,0548 *$ & $0,3085^{*}$ & $0,4546^{*}$ \\
\hline 2009 & $0,3594 *$ & $0,0530 *$ & $0,2955^{*}$ & $0,4654^{*}$ \\
\hline
\end{tabular}

Fuente: elaboración propia.

Nota: * indica valores significativos al $1 \%$

\section{Bibliografía}

Almås, I. (2008), "Equalizing income versus equalizing opportunity: a comparison of the United States and Germany", Research on Economic Inequality, vol. 16, Emerald.

Almås, I. y otros (2011), "Measuring unfair (in)equality", Journal of Public Economics, vol. 95, $\mathrm{N}^{\circ}$ 7-8, Amsterdam, Elsevier.

Arneson, R. (1989), "Equality and equal opportunity for welfare", Philosophical Studies, vol. 56, $\mathrm{N}^{\circ} 1$, Springer.

Assis, R.S. de, E.M. Costa y J.L. Mariano (2012), "Impacto da migração de não naturais e da migração de retorno sobre a distribuição de renda dos estados da Bahia e de São Paulo: um olhar sobre a inserção desses indivíduos no mercado de trabalho local", $40^{\circ}$ Encontro Nacional de Economia [en línea] http://www.anpec.org.br/novosite/br/encontro-2012.

Azevedo, J. (2007), "Avaliando a significância estatística da queda na desigualdade no Brasil", Desigualdade de renda no Brasil: uma análise da queda recente, R. Barros, M. Foguel y G. Ullysea (orgs.), Brasilia, Instituto de Investigación Económica Aplicada (IPEA).

Barros, R.P. de, M. Carvalho y S. Franco (2006), "A efetividade do salário mínimo como um instrumento para reduzir a pobreza no Brasil", Boletim de Conjuntura, $\mathrm{N}^{\circ} 74$, Instituto de Investigación Económica Aplicada (IPEA).

Betts, J. y J. Roemer (2005), "Equalizing Opportunity for Racial and Socioeconomic Groups in the United States through Educational Finance Reform" [en línea] http://www. escholarship.org/uc/item/0gq4z4m9\#page-3.

Bourguignon, F., F. Ferreira y M. Menéndez (2007), "Inequality of opportunity in Brazil", Review of Income and Wealth, vol. 53, $\mathrm{N}^{\circ} 4$, Wiley.

Checchi, D. y V. Peragine (2009), "Regional disparities and inequality of opportunity: the case of Italy", Journal of Economic Inequality, por aparecer.

Devooght, K. (2008), "To each the same and to each his own: a proposal to measure responsibility-sensitive income inequality", Economica, vol. 75, $\mathrm{N}^{\circ} 298$.
Dworkin, R. (1981), "What is equality? Part 2: equality of resources", Philosophy and Public Affairs, vol. 10, $\mathrm{N}^{\circ} 4$, Princeton, Princeton University Press.

Ferreira, F. y otros (2006), "Ascensão e queda da desgiualdade de renda no Brasil", Económica, vol. 8, $\mathrm{N}^{\circ} 1$.

Figueiredo, E. y J.L. Netto Júnior (2014), "More equal but not so fair: an analysis of Brazilian income distribution from 1995 to 2009", Empirical Economics, vol. 46, N 4, Springer.

Fleurbaey, M. (1998), "Equality among responsible individuals", Freedom in Economics: New Perspectives in Normative Analysis, J.-F. Laslier y otros (eds.), Londres, Routledge.

Fleurbaey, M. y F. Maniquet (2011), "Compensation and responsibility", Handbook of Social Choice and Welfare, vol. II, K. Arrow, S. Sen y K. Suzumura (eds.), Amsterdam, Elsevier

Giambiagi, F. y S. Franco (2007), "O esgotamento do papel do salário mínimo como mecanismo de combate à pobreza extrema", Texto para Discussão, $\mathrm{N}^{\circ} 1290$, Río de Janeiro, Instituto de Investigación Económica Aplicada (IPEA).

Granger, C., E. Maasoumi y J. Racine (2004), "A dependence metric for possibly nonlinear processes", Journal of Time Series Analysis, vol. 25, $\mathrm{N}^{\circ} 5$, Wiley.

Härdle, W. (1990), Applied Nonparametric Regression, Cambridge, Cambridge University Press.

Kranich, L. (1996), "Equitable opportunities: an axiomatic approach", Journal of Economic Theory, vol. 71, $\mathrm{N}^{\circ} 1$, Amsterdam, Elsevier.

Li, Q. y J. Racine (2007), Nonparametric Econometrics: Theory and Practice, Princeton, Princeton University Press.

Li, Q., E. Maasoumi y J.S. Racine (2009), "A nonparametric test for equality of distributions with mixed categorical and continuous data", Journal of Econometrics, vol. 148, $\mathrm{N}^{\circ} 2$, Amsterdam, Elsevier.

Maasoumi, E., J. Racine y T. Stengos (2007), "Growth and convergence: a profile of distribution dynamics and mobility", Journal of Econometrics, vol. 136, $\mathrm{N}^{\circ} 2$, Amsterdam, Elsevier. 
Machado de Assis, J. (1891), Quincas Borba, Río de Janeiro, B.L. Garnier, Livrero-Editor.

Maloney, W.F. (2000), "A note on minimum wages in Latin America", Washington, D.C., Banco Mundial.

Menezes-Filho, N. (2001), "A evolução da educação no Brasil e seu impacto no mercado de trabalho", Instituto Futuro Brasil, São Paulo, marzo [en línea] http://www.anj.org.br/pje/biblioteca/ publicacoes/A\%20Evolucao\%20da\%20educacao\%20no\%20 Brasil\%20e\%20seu\%20impacto\%20no\%20Mercado\%20 de\%20trabalho.pdf.

Menezes-Filho, N., R. Fernandes y P. Picchetti (2006), "Rising human capital but constant inequality: the education composition effect in Brazil", Revista Brasileira de Economia, vol. 60, $\mathrm{N}^{\circ} 4$, Río de Janeiro.

Menezes-Filho, N., M. Mendes y S.E. de Almeida (2004), "O diferencial de salários formal-informal no Brasil: segmentação ou viés de seleção?", Revista Brasileira de Economia, vol. 58, $\mathrm{N}^{\circ} 2$, Río de Janeiro.

Menezes-Filho, N., E. Rodrigues y A. de Souza. (2009), "Salário mínimo e desigualdade no Brasil entre 1981-1999: uma abordagem semiparamétrica", Revista Brasileira de Economia, vol. 63, $\mathrm{N}^{\circ}$ 3, Río de Janeiro.

O'Neill, D., O. Sweetman y D. Van de Gaer (2001), "Equality of opportunity and kernel density estimation: an application to intergenerational mobility", Applying Kernel and Nonparametric Estimation to Economic Topics, vol. 14, Stanford, JAI Press.

Ooghe, E., E. Schokkaert y D. Van de Gaer (2007), "Equality of opportunity versus equality of opportunity sets", Social Choice and Welfare, vol. $28, \mathrm{~N}^{\circ} 2$, Springer.
Pastore, J. y N. Silva (2000), Mobilidade social no Brasil, São Paulo, Macron Books.

Pattanaik, P. e Y. Xu (1990), "On ranking opportunity sets in terms of freedom of choice", Recherches Économiques de Louvain, vol. 56, $\mathrm{N}^{\circ} 3-4$, Universidad Católica de Lovaina.

Racine, J. (2006), "Consistent specification testing of heteroskedastic parametric regression quantile models with mixed data", inédito.

Racine, J.S. y Q. Li (2004), "Nonparametric estimation of regression functions with both categorical and continuous data", Journal of Econometrics, vol. 119, $\mathrm{N}^{\circ} 1$, Amsterdam, Elsevier.

Roemer, J. (1998), Equality of Opportunity, Nueva York, Harvard University Press.

(1996), Theories of Distributive Justice, Cambridge, Massachusetts, Harvard University Press.

Saboia, J. (2006), "Salário mínimo e combate à pobreza", Valor Econômico.

Salvi, A. (2007), "An empirical approach to the measurement of equality of opportunity", Milán, Università degli Studi di Milano, inédito.

Soares, S. (2006), "Distribuição de renda no Brasil de 1976 a 2004 com ênfase no período entre 2001 e 2004", Texto para discussão, $\mathrm{N}^{\circ} 1166$, Río de Janeiro, Instituto de Investigación Económica Aplicada (IPEA).

Thomson, W. (2011), "Fair allocation rules", Handbook of Social Choice and Welfare, vol. 2, K. Arrow, A. Sen y K. Suzumura (eds.), Amsterdam, Elsevier. 